\title{
Search for Anomalous Top-Quark Couplings with the D0 Detector
}

V. M. Abazov, ${ }^{36}$ B. Abbott,${ }^{75}$ M. Abolins, ${ }^{65}$ B. S. Acharya, ${ }^{29}$ M. Adams, ${ }^{51}$ T. Adams,${ }^{49}$ E. Aguilo, ${ }^{6}$ M. Ahsan, ${ }^{59}$ G. D. Alexeev, ${ }^{36}$ G. Alkhazov, ${ }^{40}$ A. Alton, ${ }^{64, *}$ G. Alverson, ${ }^{63}$ G. A. Alves, ${ }^{2}$ M. Anastasoaie, ${ }^{35}$ L. S. Ancu, ${ }^{35}$ T. Andeen, ${ }^{53}$ B. Andrieu, ${ }^{17}$ M. S. Anzelc, ${ }^{53}$ M. Aoki,${ }^{50}$ Y. Arnoud, ${ }^{14}$ M. Arov,${ }^{60}$ M. Arthaud, ${ }^{18}$ A. Askew, ${ }^{49, \dagger}$ B. Åsman,${ }^{41}$ A.C. S. Assis Jesus, ${ }^{3}$ O. Atramentov, ${ }^{49}$ C. Avila,${ }^{8}$ J. BackusMayes,${ }^{82}$ F. Badaud,${ }^{13}$ L. Bagby, ${ }^{50}$ B. Baldin,${ }^{50}$ D. V. Bandurin, ${ }^{59}$ P. Banerjee, ${ }^{29}$ S. Banerjee,${ }^{29}$ E. Barberis, ${ }^{63}$ A.-F. Barfuss, ${ }^{15}$ P. Bargassa, ${ }^{80}$ P. Baringer,${ }^{58}$ J. Barreto, ${ }^{2}$ J. F. Bartlett ${ }^{50}$ U. Bassler, ${ }^{18}$ D. Bauer, ${ }^{43}$ S. Beale, ${ }^{6}$ A. Bean,${ }^{58}$ M. Begalli, ${ }^{3}$ M. Begel,${ }^{73}$ C. Belanger-Champagne, ${ }^{41}$ L. Bellantoni, ${ }^{50}$ A. Bellavance, ${ }^{50}$ J. A. Benitez, ${ }^{65}$ S. B. Beri, ${ }^{27}$ G. Bernardi,${ }^{17}$ R. Bernhard,${ }^{23}$ I. Bertram, ${ }^{42}$ M. Besançon, ${ }^{18}$ R. Beuselinck, ${ }^{43}$ V. A. Bezzubov,${ }^{39}$ P. C. Bhat,${ }^{50}$ V. Bhatnagar ${ }^{27}$ G. Blazey,${ }^{52}$ F. Blekman, ${ }^{43}$ S. Blessing, ${ }^{49}$ K. Bloom, ${ }^{67}$ A. Boehnlein, ${ }^{50}$ D. Boline, ${ }^{62}$ T. A. Bolton, ${ }^{59}$ E. E. Boos,${ }^{38}$ G. Borissov, ${ }^{42}$ T. Bose, ${ }^{77}$ A. Brandt ${ }^{78}$ R. Brock, ${ }^{65}$ G. Brooijmans, ${ }^{70}$ A. Bross ${ }^{50}$ D. Brown, ${ }^{19}$ X. B. Bu, ${ }^{7}$ N. J. Buchanan, ${ }^{49}$ D. Buchholz,${ }^{53}$ M. Buehler, ${ }^{81}$ V. Buescher, ${ }^{22}$ V. Bunichev, ${ }^{38}$ S. Burdin, ${ }^{42,}$ T. H. Burnett ${ }^{82}$ C. P. Buszello, ${ }^{43}$ P. Calfayan, ${ }^{25}$ B. Calpas, ${ }^{15}$ S. Calvet, ${ }^{16}$ J. Cammin,${ }^{71}$ M. A. Carrasco-Lizarraga, ${ }^{33}$ E. Carrera, ${ }^{49}$ W. Carvalho, ${ }^{3}$ B. C. K. Casey, ${ }^{50}$ H. Castilla-Valdez, ${ }^{33}$ S. Chakrabarti, ${ }^{72}$ D. Chakraborty, ${ }^{52}$ K. M. Chan, ${ }^{55}$ A. Chandra, ${ }^{48}$ E. Cheu, ${ }^{45}$ D. K. Cho, ${ }^{62}$ S. Choi, ${ }^{32}$ B. Choudhary, ${ }^{28}$ L. Christofek,${ }^{77}$ T. Christoudias, ${ }^{43}$ S. Cihangir, ${ }^{50}$ D. Claes,${ }^{67}$ J. Clutter, ${ }^{58}$ M. Cooke, ${ }^{50}$ W. E. Cooper, ${ }^{50}$ M. Corcoran, ${ }^{80}$ F. Couderc,${ }^{18}$ M.-C. Cousinou, ${ }^{15}$ S. Crépé-Renaudin,,${ }^{14}$ V. Cuplov,${ }^{59}$ D. Cutts, ${ }^{77}$ M. Ćwiok, ${ }^{30}$ H. da Motta, ${ }^{2}$ A. Das, ${ }^{45}$ G. Davies, ${ }^{43}$ K. De,${ }^{78}$ S. J. de Jong, ${ }^{35}$ E. De La Cruz-Burelo, ${ }^{33}$ C. De Oliveira Martins, ${ }^{3}$ K. DeVaughan,${ }^{67}$ F. Déliot,${ }^{18}$ M. Demarteau ${ }^{50}$ R. Demina, ${ }^{71}$ D. Denisov ${ }^{50}$ S. P. Denisov, ${ }^{39}$ S. Desai,${ }^{50}$ H. T. Diehl,${ }^{50}$ M. Diesburg, ${ }^{50}$ A. Dominguez,${ }^{67}$ T. Dorland,${ }^{82}$ A. Dubey, ${ }^{28}$ L. V. Dudko, ${ }^{38}$ L. Duflot, ${ }^{16}$ S. R. Dugad,${ }^{29}$ D. Duggan, ${ }^{49}$ A. Duperrin, ${ }^{15}$ S. Dutt, ${ }^{27}$ J. Dyer, ${ }^{65}$ A. Dyshkant,${ }^{52}$ M. Eads, ${ }^{67}$ D. Edmunds, ${ }^{65}$ J. Ellison, ${ }^{48}$ V. D. Elvira, ${ }^{50}$ Y. Enari, ${ }^{77}$ S. Eno, ${ }^{61}$ P. Ermolov, ${ }^{38,} \S$ M. Escalier, ${ }^{15}$ H. Evans,${ }^{54}$ A. Evdokimov, ${ }^{73}$ V. N. Evdokimov, ${ }^{39}$ A. V. Ferapontov, ${ }^{59}$ T. Ferbel,,${ }^{61,71}$ F. Fiedler, ${ }^{24}$ F. Filthaut, ${ }^{35}$ W. Fisher, ${ }^{50}$ H. E. Fisk,${ }^{50}$ M. Fortner, ${ }^{52}$ H. Fox ${ }^{42}$ S. Fu, ${ }^{50}$ S. Fuess, ${ }^{50}$ T. Gadfort, ${ }^{70}$ C. F. Galea, ${ }^{35}$ C. Garcia, ${ }^{71}$ A. Garcia-Bellido, ${ }^{71}$ V. Gavrilov, ${ }^{37}$ P. Gay ${ }^{13}$ W. Geist, ${ }^{19}$ W. Geng, ${ }^{15,65}$ C. E. Gerber, ${ }^{51}$ Y. Gershtein, ${ }^{49, \dagger}$ D. Gillberg, ${ }^{6}$ G. Ginther, ${ }^{71}$ B. Gómez, ${ }^{8}$ A. Goussiou, ${ }^{82}$

P. D. Grannis, ${ }^{72}$ H. Greenlee, ${ }^{50}$ Z. D. Greenwood ${ }^{60}$ E. M. Gregores ${ }^{4}$ G. Grenier,${ }^{20}$ Ph. Gris, ${ }^{13}$ J.-F. Grivaz, ${ }^{16}$

A. Grohsjean, ${ }^{25}$ S. Grünendahl,${ }^{50}$ M. W. Grünewald, ${ }^{30}$ F. Guo, ${ }^{72}$ J. Guo, ${ }^{72}$ G. Gutierrez, ${ }^{50}$ P. Gutierrez, ${ }^{75}$ A. Haas, ${ }^{70}$

N. J. Hadley, ${ }^{61}$ P. Haefner, ${ }^{25}$ S. Hagopian, ${ }^{49}$ J. Haley, ${ }^{68}$ I. Hall, ${ }^{65}$ R. E. Hall,${ }^{47}$ L. Han, ${ }^{7}$ K. Harder,${ }^{44}$ A. Harel,${ }^{71}$ J. M. Hauptman, ${ }^{57}$ J. Hays, ${ }^{43}$ T. Hebbeker, ${ }^{21}$ D. Hedin, ${ }^{52}$ J. G. Hegeman, ${ }^{34}$ A. P. Heinson, ${ }^{48}$ U. Heintz, ${ }^{62}$ C. Hensel, ${ }^{22,8}$ K. Herner, ${ }^{72}$ G. Hesketh, ${ }^{63}$ M. D. Hildreth, ${ }^{55}$ R. Hirosky, ${ }^{81}$ T. Hoang, ${ }^{49}$ J. D. Hobbs ${ }^{72}$ B. Hoeneisen, ${ }^{12}$ M. Hohlfeld, ${ }^{22}$ S. Hossain, ${ }^{75}$ P. Houben, ${ }^{34}$ Y. Hu, ${ }^{72}$ Z. Hubacek,,${ }^{10}$ N. Huske, ${ }^{17}$ V. Hynek, ${ }^{9}$ I. Iashvili, ${ }^{69}$ R. Illingworth,${ }^{50}$ A. S. Ito, ${ }^{50}$ S. Jabeen, ${ }^{62}$ M. Jaffré, ${ }^{16}$ S. Jain, ${ }^{75}$ K. Jakobs, ${ }^{23}$ C. Jarvis, ${ }^{61}$ R. Jesik ${ }^{43}$ K. Johns,${ }^{45}$ C. Johnson, ${ }^{70}$ M. Johnson, ${ }^{50}$ D. Johnston, ${ }^{67}$ A. Jonckheere, ${ }^{50}$ P. Jonsson, ${ }^{43}$ A. Juste, ${ }^{50}$ E. Kajfasz, ${ }^{15}$ D. Karmanov, ${ }^{38}$ P. A. Kasper, ${ }^{50}$ I. Katsanos, ${ }^{70}$ V. Kaushik ${ }^{78}$ R. Kehoe, ${ }^{79}$ S. Kermiche, ${ }^{15}$ N. Khalatyan, ${ }^{50}$ A. Khanov,${ }^{76}$ A. Kharchilava, ${ }^{69}$ Y. N. Kharzheev, ${ }^{36}$ D. Khatidze, ${ }^{70}$ T. J. Kim, ${ }^{31}$ M. H. Kirby, ${ }^{53}$ M. Kirsch, ${ }^{21}$ B. Klima, ${ }^{50}$ J. M. Kohli, ${ }^{27}$ J.-P. Konrath, ${ }^{23}$ A. V. Kozelov, ${ }^{39}$

J. Kraus, ${ }^{65}$ T. Kuhl, ${ }^{24}$ A. Kumar, ${ }^{69}$ A. Kupco, ${ }^{11}$ T. Kurča,${ }^{20}$ V. A. Kuzmin, ${ }^{38}$ J. Kvita, ${ }^{9}$ F. Lacroix, ${ }^{13}$ D. Lam, ${ }^{55}$ S. Lammers, ${ }^{70}$ G. Landsberg, ${ }^{77}$ P. Lebrun, ${ }^{20}$ W. M. Lee, ${ }^{50}$ A. Leflat, ${ }^{38}$ J. Lellouch, ${ }^{17}$ J. Li $,{ }^{78,8 \S} \mathrm{L} . \mathrm{Li},{ }^{48}$ Q. Z. Li,${ }^{50}$ S. M. Lietti, ${ }^{5}$ J. K. Lim, ${ }^{31}$ J. G. R. Lima, ${ }^{52}$ D. Lincoln,${ }^{50}$ J. Linnemann,${ }^{65}$ V. V. Lipaev, ${ }^{39}$ R. Lipton, ${ }^{50}$ Y. Liu, ${ }^{7}$ Z. Liu, ${ }^{6}$ A. Lobodenko, ${ }^{40}$ M. Lokajicek, ${ }^{11}$ P. Love, ${ }^{42}$ H. J. Lubatti, ${ }^{82}$ R. Luna-Garcia, ${ }^{33, \|}$ A. L. Lyon, ${ }^{50}$ A. K. A. Maciel, ${ }^{2}$ D. Mackin, ${ }^{80}$ R. J. Madaras, ${ }^{46}$ P. Mättig, ${ }^{26}$ A. Magerkurth, ${ }^{64}$ P. K. Mal, ${ }^{82}$ H. B. Malbouisson, ${ }^{3}$ S. Malik, ${ }^{67}$ V. L. Malyshev, ${ }^{36}$ Y. Maravin, ${ }^{59}$ B. Martin, ${ }^{14}$ R. McCarthy, ${ }^{72}$ M. M. Meijer, ${ }^{35}$ A. Melnitchouk, ${ }^{66}$ L. Mendoza, ${ }^{8}$ P. G. Mercadante, ${ }^{5}$ M. Merkin, ${ }^{38}$ K. W. Merritt, ${ }^{50}$ A. Meyer, ${ }^{21}$ J. Meyer, ${ }^{22,8}$ J. Mitrevski, ${ }^{70}$ R. K. Mommsen,${ }^{44}$ N. K. Mondal, ${ }^{29}$ R. W. Moore, ${ }^{6}$ T. Moulik, ${ }^{58}$ G. S. Muanza, ${ }^{15}$ M. Mulhearn, ${ }^{70}$ O. Mundal, ${ }^{22}$ L. Mundim, ${ }^{3}$ E. Nagy, ${ }^{15}$ M. Naimuddin,${ }^{50}$ M. Narain, ${ }^{77}$ H. A. Neal, ${ }^{64}$ J. P. Negret ${ }^{8}$ P. Neustroev, ${ }^{40}$ H. Nilsen, ${ }^{23}$ H. Nogima, ${ }^{3}$ S. F. Novaes, ${ }^{5}$ T. Nunnemann, ${ }^{25}$ D. C. O'Neil, ${ }^{6}$ G. Obrant,${ }^{40}$ C. Ochando, ${ }^{16}$ D. Onoprienko, ${ }^{59}$ N. Oshima, ${ }^{50}$ N. Osman, ${ }^{43}$ J. Osta, ${ }^{55}$ R. Otec, ${ }^{10}$ G. J. Otero y Garzón, ${ }^{1}$

M. Owen ${ }^{44}$ M. Padilla, ${ }^{48}$ P. Padley, ${ }^{80}$ M. Pangilinan, ${ }^{77}$ N. Parashar, ${ }^{56}$ S.-J. Park,${ }^{22,8}$ S. K. Park, ${ }^{31}$ J. Parsons, ${ }^{70}$

R. Partridge,${ }^{77}$ N. Parua,${ }^{54}$ A. Patwa,${ }^{73}$ G. Pawloski ${ }^{80}$ B. Penning,${ }^{23}$ M. Perfilov,${ }^{38}$ K. Peters,${ }^{44}$ Y. Peters,${ }^{26}$ P. Pétroff, ${ }^{16}$ M. Petteni, ${ }^{43}$ R. Piegaia, ${ }^{1}$ J. Piper, ${ }^{65}$ M.-A. Pleier ${ }^{22}$ P. L. M. Podesta-Lerma,${ }^{33, \pi l}$ V. M. Podstavkov, ${ }^{50}$ Y. Pogorelov, ${ }^{55}$ M.-E. Pol, ${ }^{2}$ P. Polozov, ${ }^{37}$ B. G. Pope,${ }^{65}$ A. V. Popov,${ }^{39}$ C. Potter,${ }^{6}$ W. L. Prado da Silva, ${ }^{3}$ H. B. Prosper, ${ }^{49}$ S. Protopopescu,${ }^{73}$ J. Qian, ${ }^{64}$ A. Quadt, ${ }^{22,8}$ B. Quinn, ${ }^{66}$ A. Rakitine, ${ }^{42}$ M. S. Rangel, ${ }^{2}$ K. Ranjan ${ }^{28}$ P. N. Ratoff, ${ }^{42}$ P. Renkel ${ }^{79}$ P. Rich, ${ }^{44}$ M. Rijssenbeek ${ }^{72}$ I. Ripp-Baudot, ${ }^{19}$ F. Rizatdinova,${ }^{76}$ S. Robinson, ${ }^{43}$ R. F. Rodrigues, ${ }^{3}$ M. Rominsky, ${ }^{75}$ C. Royon, ${ }^{18}$ 
P. Rubinov, ${ }^{50}$ R. Ruchti, ${ }^{55}$ G. Safronov,${ }^{37}$ G. Sajot,${ }^{14}$ A. Sánchez-Hernández,${ }^{33}$ M. P. Sanders, ${ }^{17}$ B. Sanghi,${ }^{50}$ G. Savage, ${ }^{50}$ L. Sawyer, ${ }^{60}$ T. Scanlon, ${ }^{43}$ D. Schaile, ${ }^{25}$ R. D. Schamberger ${ }^{72}$ Y. Scheglov, ${ }^{40}$ H. Schellman, ${ }^{53}$ T. Schliephake, ${ }^{26}$ S. Schlobohm, ${ }^{82}$ C. Schwanenberger, ${ }^{44}$ R. Schwienhorst ${ }^{65}$ J. Sekaric, ${ }^{49}$ H. Severini,${ }^{75}$ E. Shabalina,${ }^{51}$ M. Shamim, ${ }^{59}$ V. Shary, ${ }^{18}$ A. A. Shchukin, ${ }^{39}$ R. K. Shivpuri, ${ }^{28}$ V. Siccardi,${ }^{19}$ V. Simak, ${ }^{10}$ V. Sirotenko, ${ }^{50}$ P. Skubic, ${ }^{75}$ P. Slattery, ${ }^{71}$ D. Smirnov, ${ }^{55}$ G. R. Snow, ${ }^{67}$ J. Snow,${ }^{74}$ S. Snyder, ${ }^{73}$ S. Söldner-Rembold, ${ }^{44}$ L. Sonnenschein, ${ }^{17}$ A. Sopczak, ${ }^{42}$ M. Sosebee, ${ }^{78}$ K. Soustruznik, ${ }^{9}$ B. Spurlock, ${ }^{78}$ J. Stark, ${ }^{14}$ V. Stolin, ${ }^{37}$ D. A. Stoyanova, ${ }^{39}$ J. Strandberg, ${ }^{64}$ S. Strandberg, ${ }^{41}$ M. A. Strang, ${ }^{69}$ E. Strauss, ${ }^{72}$ M. Strauss, ${ }^{75}$ R. Ströhmer, ${ }^{25}$ D. Strom,${ }^{53}$ L. Stutte,${ }^{50}$ S. Sumowidagdo, ${ }^{49}$ P. Svoisky, ${ }^{35}$ A. Sznajder, ${ }^{3}$ A. Tanasijczuk, ${ }^{1}$ W. Taylor, ${ }^{6}$ B. Tiller, ${ }^{25}$ F. Tissandier, ${ }^{13}$ M. Titov, ${ }^{18}$ V. V. Tokmenin,${ }^{36}$ I. Torchiani, ${ }^{23}$ D. Tsybychev, ${ }^{72}$ B. Tuchming, ${ }^{18}$ C. Tully, ${ }^{68}$ P. M. Tuts, ${ }^{70}$ R. Unalan, ${ }^{65}$ L. Uvarov, ${ }^{40}$ S. Uvarov,${ }^{40}$ S. Uzunyan,${ }^{52}$ B. Vachon, ${ }^{6}$ P. J. van den Berg, ${ }^{34}$ R. Van Kooten,${ }^{54}$ W. M. van Leeuwen, ${ }^{34}$ N. Varelas, ${ }^{51}$ E. W. Varnes, ${ }^{45}$ I. A. Vasilyev, ${ }^{39}$ P. Verdier, ${ }^{20}$ L. S. Vertogradov, ${ }^{36} \mathrm{M}$. Verzocchi, ${ }^{50} \mathrm{D}$. Vilanova, ${ }^{18} \mathrm{~F}$. Villeneuve-Seguier, ${ }^{43} \mathrm{P}$. Vint, ${ }^{43}$ P. Vokac, ${ }^{10} \mathrm{M}$. Voutilainen, ${ }^{67, * *}$ R. Wagner, ${ }^{68}$ H. D. Wahl, ${ }^{49}$ M. H. L. S. Wang, ${ }^{50}$ J. Warchol, ${ }^{55}$ G. Watts, ${ }^{82}$ M. Wayne, ${ }^{55}$ G. Weber, ${ }^{24}$ M. Weber, ${ }^{50, \dagger \dagger}$ L. Welty-Rieger, ${ }^{54}$ A. Wenger ${ }^{23, * *}$ N. Wermes,${ }^{22}$ M. Wetstein, ${ }^{61}$ A. White, ${ }^{78}$ D. Wicke, ${ }^{26}$ M. R. J. Williams,${ }^{42}$ G. W. Wilson, ${ }^{58}$ S. J. Wimpenny, ${ }^{48}$ M. Wobisch, ${ }^{60}$ D. R. Wood, ${ }^{63}$ T. R. Wyatt, ${ }^{44}$ Y. Xie, ${ }^{77}$ C. Xu, ${ }^{64}$ S. Yacoob, ${ }^{53}$ R. Yamada, ${ }^{50}$ W.-C. Yang, ${ }^{44}$ T. Yasuda,${ }^{50}$ Y. A. Yatsunenko, ${ }^{36}$ Z. Ye,${ }^{50}$ H. Yin, ${ }^{7}$ K. Yip, ${ }^{73}$ H. D. Yoo, ${ }^{77}$ S. W. Youn, ${ }^{53}$ J. Yu, ${ }^{78}$ C. Zeitnitz, ${ }^{26}$ S. Zelitch, ${ }^{81}$ T. Zhao, ${ }^{82}$ B. Zhou, ${ }^{64}$ J. Zhu, ${ }^{72}$ M. Zielinski, ${ }^{71}$ D. Zieminska, ${ }^{54}$ L. Zivkovic ${ }^{70}$ V. Zutshi, ${ }^{52}$ and E. G. Zverev ${ }^{38}$

(The D0 Collaboration)

\author{
${ }^{1}$ Universidad de Buenos Aires, Buenos Aires, Argentina \\ ${ }^{2}$ LAFEX, Centro Brasileiro de Pesquisas Físicas, Rio de Janeiro, Brazil \\ ${ }^{3}$ Universidade do Estado do Rio de Janeiro, Rio de Janeiro, Brazil \\ ${ }^{4}$ Universidade Federal do ABC, Santo André, Brazil \\ ${ }^{5}$ Instituto de Física Teórica, Universidade Estadual Paulista, São Paulo, Brazil \\ ${ }^{6}$ University of Alberta, Edmonton, Alberta, Canada, \\ Simon Fraser University, Burnaby, British Columbia, Canada, \\ York University, Toronto, Ontario, Canada, \\ and McGill University, Montreal, Quebec, Canada \\ ${ }^{7}$ University of Science and Technology of China, Hefei, People's Republic of China \\ ${ }^{8}$ Universidad de los Andes, Bogotá, Colombia \\ ${ }^{9}$ Center for Particle Physics, Charles University, Prague, Czech Republic \\ ${ }^{10}$ Czech Technical University, Prague, Czech Republic \\ ${ }^{11}$ Center for Particle Physics, Institute of Physics, Academy of Sciences of the Czech Republic, Prague, Czech Republic \\ ${ }^{12}$ Universidad San Francisco de Quito, Quito, Ecuador \\ ${ }^{13}$ LPC, Université Blaise Pascal, CNRS/IN2P3, Clermont, France \\ ${ }^{14}$ LPSC, Université Joseph Fourier Grenoble 1, CNRS/IN2P3, Institut National Polytechnique de Grenoble, Grenoble, France \\ ${ }^{15}$ CPPM, Aix-Marseille Université, CNRS/IN2P3, Marseille, France \\ ${ }^{16}$ LAL, Université Paris-Sud, IN2P3/CNRS, Orsay, France \\ ${ }^{17}$ LPNHE, IN2P3/CNRS, Universités Paris VI and VII, Paris, France \\ ${ }^{18}$ CEA, Irfu, SPP, Saclay, France \\ ${ }^{19}$ IPHC, Université Louis Pasteur, CNRS/IN2P3, Strasbourg, France \\ ${ }^{20} I P N L$, Université Lyon 1, CNRS/IN2P3, Villeurbanne, France and Université de Lyon, Lyon, France \\ ${ }^{21}$ III. Physikalisches Institut A, RWTH Aachen University, Aachen, Germany \\ ${ }^{22}$ Physikalisches Institut, Universität Bonn, Bonn, Germany \\ ${ }^{23}$ Physikalisches Institut, Universität Freiburg, Freiburg, Germany \\ ${ }^{24}$ Institut für Physik, Universität Mainz, Mainz, Germany \\ ${ }^{25}$ Ludwig-Maximilians-Universität München, München, Germany \\ ${ }^{26}$ Fachbereich Physik, University of Wuppertal, Wuppertal, Germany \\ ${ }^{27}$ Panjab University, Chandigarh, India \\ ${ }^{28}$ Delhi University, Delhi, India \\ ${ }^{29}$ Tata Institute of Fundamental Research, Mumbai, India \\ ${ }^{30}$ University College Dublin, Dublin, Ireland \\ ${ }^{31}$ Korea Detector Laboratory, Korea University, Seoul, Korea \\ ${ }^{32}$ SungKyunKwan University, Suwon, Korea \\ ${ }^{33}$ CINVESTAV, Mexico City, Mexico \\ ${ }^{34}$ FOM-Institute NIKHEF and University of Amsterdam/NIKHEF, Amsterdam, The Netherlands
}




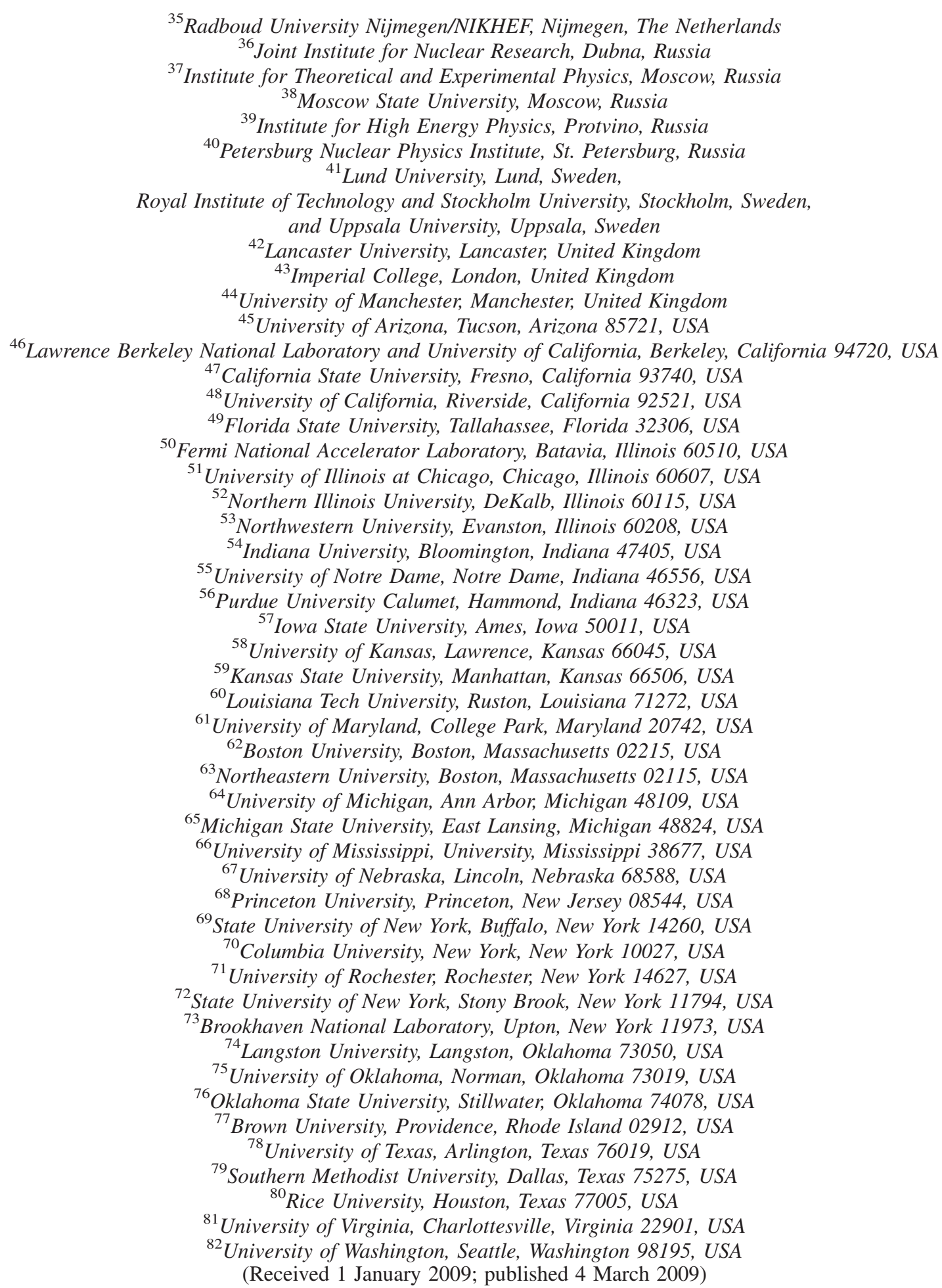

Anomalous $W t b$ couplings modify the angular correlations of the top-quark decay products and change the single top-quark production cross section. We present limits on anomalous top-quark couplings by combining information from $W$ boson helicity measurements in top-quark decays and anomalous coupling searches in the single top-quark final state. We set limits on right-handed vector couplings as well as left-handed and right-handed tensor couplings based on about $1 \mathrm{fb}^{-1}$ of data collected by the D0 experiment. 
The top quark is by far the heaviest fermion in the standard model (SM), and thus has the strongest coupling to the Higgs boson of all SM fermions. This makes the top quark and its interactions an ideal place to look for new physics related to electroweak symmetry breaking. The coupling between the top quark and the weak gauge bosons may be altered by physics beyond the SM. In particular, the coupling between the top quark and the $W$ boson determines most of the top-quark phenomenology and can be sensitively probed at hadron colliders [1]. The effective Lagrangian describing the $W t b$ interaction including operators up to dimension five is [2]

$$
\begin{aligned}
\mathcal{L}= & -\frac{g}{\sqrt{2}} \bar{b} \gamma^{\mu} V_{t b}\left(f_{1}^{L} P_{L}+f_{1}^{R} P_{R}\right) t W_{\mu}^{-} \\
& -\frac{g}{\sqrt{2}} \bar{b} \frac{i \sigma^{\mu \nu} q_{\nu} V_{t b}}{M_{W}}\left(f_{2}^{L} P_{L}+f_{2}^{R} P_{R}\right) t W_{\mu}^{-}+\text {H.c. }
\end{aligned}
$$

where $M_{W}$ is the mass of the $W$ boson, $q_{\nu}$ is its fourmomentum, $V_{t b}$ is the Cabibbo-Kobayashi-Maskawa matrix element [3], and $P_{L}=\left(1-\gamma_{5}\right) / 2\left[P_{R}=\left(1+\gamma_{5}\right) / 2\right]$ is the left-handed (right-handed) projection operator. In the $\mathrm{SM}$, the $W t b$ coupling is purely left-handed, and the values of the coupling form factors are $f_{1}^{L} \approx 1, f_{2}^{L}=f_{1}^{R}=f_{2}^{R}=$ 0 . We assume real coupling form factors, implying $C P$ conservation, and a spin- $\frac{1}{2}$ top quark which decays predominantly to $W b$. Indirect constraints on the magnitude of the right-handed vector coupling and tensor couplings exist from measurements of the $b \rightarrow s \gamma$ branching fraction [4]. While those limits are tighter than the direct limits presented here, they also include assumptions that are not required here, for example, the absence of other sources of new physics coupling to the $b$ quark.

We search for non-SM values of the couplings using $\approx 1 \mathrm{fb}^{-1}$ of data collected by the D0 experiment [5] at the Fermilab Tevatron $p \bar{p}$ collider between 2002 and 2006 (Run II). Variations in the coupling form factors would mainly manifest themselves in two distinct ways at D0: by changing the rate and kinematic distributions of electroweak single top-quark production and by altering the fractions of $W$ bosons from top-quark decays produced in each of the three possible helicity states. In this Letter, we combine information from our measurement of the $W$ boson helicity fractions in $t \bar{t}$ events [6] with information from single top-quark production. We have previously set direct limits on anomalous top-quark coupling form factors based solely on the single top-quark final state [7]. Here, we set substantially tighter limits on the effective top-quark couplings using the general framework given in Ref. [8]. This is the first such combination of all applicable D0 Run II top-quark measurements to limit anomalous top-quark coupling form factors.

We follow the approach adopted in Ref. [7] and investigate one pair of coupling form factors at a time out of the full set of form factors $\left(f_{1}^{L}, f_{1}^{R}, f_{2}^{L}\right.$, and $\left.f_{2}^{R}\right)$. For each pair under investigation, we assume that the other two have the SM values. We consider three cases, pairing the lefthanded vector coupling form factor $f_{1}^{L}$ with each of the other three form factors. We refer to these as $\left(L_{1}, R_{1}\right),\left(L_{1}\right.$, $\left.L_{2}\right)$, and $\left(L_{1}, R_{2}\right)$. For each pair of form factors, a likelihood distribution is extracted from the $W$ helicity measurement of the decay angle distribution in top-quark decays. All top-quark pair events with decays to at least one lepton (electron or muon) are included in the $W$ helicity measurement. This likelihood is then combined with the result of the anomalous couplings search in the single top-quark final state in a Bayesian statistical analysis, yielding a two-dimensional posterior probability density as a function of both form factors. We extract limits on $f_{1}^{R}, f_{2}^{L}$, and $f_{2}^{R}$ by projecting the two-dimensional posterior onto the corresponding form factor axis.

The $W$ boson helicity measurement, described in Ref. [6], uses events in both the $\ell+$ jets $(t \bar{t} \rightarrow$ $\left.W^{+} W^{-} b \bar{b} \rightarrow \ell \nu q \bar{q}^{\prime} b \bar{b}\right)$ and dilepton $\left(t \bar{t} \rightarrow W^{+} W^{-} b \bar{b} \rightarrow\right.$ $\ell \nu \ell^{\prime} \nu^{\prime} b \bar{b}$ ) final states. The measurement variable is $\theta^{*}$, the angle between the down-type fermion and top-quark momenta in the $W$ boson rest frame. To evaluate this variable, we assign a momentum to the neutrino(s) either via a constrained kinematic fit (in the $\ell+$ jets channel) or an algebraic solution (in the dilepton channel).

We use the ALPGEN leading-order Monte Carlo (MC) event generator [9], interfaced to PYTHIA [10], to model $t \bar{t}$ events as well as $W+$ jets and $Z+$ jets background events. We generate both SM $V-A$ and $V+A W t b$ couplings, and reweight events to model a given $W$ boson helicity state. We use the CTEQ6L1 parton distribution functions [11] and set the top-quark mass to $172.5 \mathrm{GeV}$. The response of the D0 detector to the MC events is simulated using GEANT [12]. We model the background from multijet production where a jet is misidentified as an isolated electron or muon using events from data containing lepton candidates which pass all of the lepton identification requirements except one but otherwise resemble the signal events. We use MC to model other small backgrounds (diboson and single top-quark production).

We select events with a multivariate likelihood discriminant that uses both kinematic and $b$-lifetime information to distinguish $t \bar{t}$ events from background and obtain a sample of $288 \ell+$ jets (75 dilepton) events with an expected background contribution of $54 \pm 7(17 \pm 4)$ events.

A binned maximum likelihood fit compares the $\cos \theta^{*}$ distribution of the selected events to the expectations for each $W$ boson helicity state plus background. We vary both the longitudinal and right-handed helicity fractions $f_{0}$ and $f_{+}$in the fit and find the relative likelihood of any set of helicity fractions being consistent with the data. In the previous $W$ helicity publication, we expressed the likelihood in terms of the helicity fractions and used a prior that was flat in $f_{0}$ and $f_{+}[6]$. Here, we instead express 
these relative likelihoods in terms of the anomalous $W t b$ coupling form factors squared using the relationships given in Ref. [8]. The resulting likelihood distributions are shown in Figs. 1(a), 1(c), and 1(e). They show that the $W$ helicity measurement only constrains ratios of the coupling form factors.

We can constrain both the ratios and the magnitudes of the form factors in the single top analysis. The dominant modes for single top-quark production at the Tevatron are the $s$-channel production and decay of a virtual $W$ boson and the $t$-channel exchange of a $W$ boson. Evidence for production of single top quarks has been reported by the D0 and CDF collaborations $[13,14]$. Both the cross section and the angular correlations of the final state objects are modified in the presence of anomalous couplings. The total cross section for SM single top-quark production at a topquark mass of $172.5 \mathrm{GeV}$ is predicted to be $3.15 \pm 0.3 \mathrm{pb}$ [15]. For this analysis, we assume that single top quarks are produced exclusively through $W$ boson exchange and that the $W t b$ vertex dominates top-quark production and decay.

We look for single top-quark production in events with one lepton [electron $\left(p_{T}>15 \mathrm{GeV}\right)$ or muon $\left(p_{T}>\right.$ $18 \mathrm{GeV})]$ and $\not_{T}>15 \mathrm{GeV}$. We select a sample that is statistically independent of the $W$ helicity analysis by asking for two or three jets with $p_{T}>15 \mathrm{GeV}$, of which one should have $p_{T}>25 \mathrm{GeV}$. We also require at least one of the jets to be identified as originating from a $b$ hadron by a $b$-tagging algorithm. Details of the selection criteria and background modeling are given in Ref. [13].

We model the single top-quark signal using the COMPHEP-SINGLETOP MC event generator [16] where anomalous $W t b$ couplings are considered in both the production and decay of the top quark. The background modeling for the single top analysis utilizes the same samples as the $W$ helicity analysis for $W+$ jets and multijet backgrounds. The $t \bar{t}$ background in the single top-quark sample is small and is modeled by simulated SM $t \bar{t}$ events. It is normalized to the theoretical cross section [17].

The selection efficiencies for single top-quark signals with different $W t b$ couplings are approximately (1-2)\% for events with one $b$ tag and less than $1 \%$ for events with two $b$ tags. We select 1152 events, which we expect to contain $56 \pm 12$ SM single top-quark events. We use boosted decision trees $[18,19]$ to extract single top-quark events from the large background.

Systematic uncertainties in the signal and background models are described in detail in Refs. [6,13]. We take all systematic uncertainties and their correlations into account. Systematic uncertainties in the $W$ boson helicity measurement arise from finite MC statistics and uncertainties on the top-quark mass, jet energy calibration, and MC models of signal and background. Variations in these parameters can change the measurement in two ways: by altering the estimate of the background in the final sample (a)

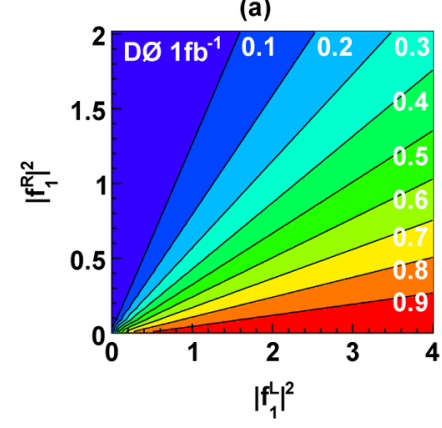

(b)

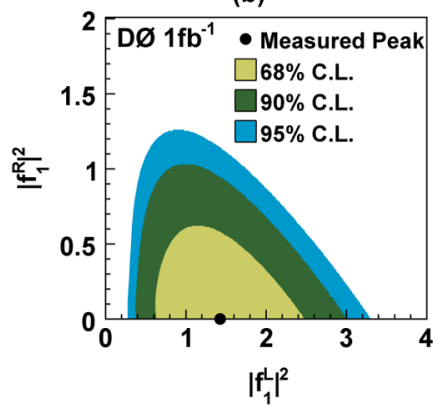

(e)

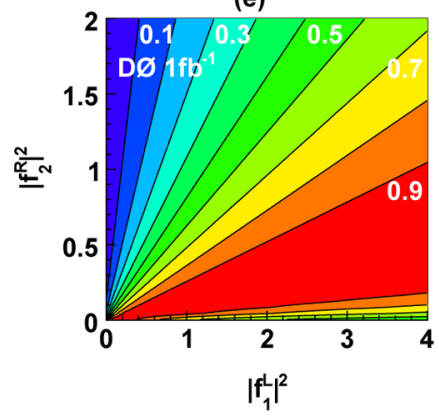

(c)
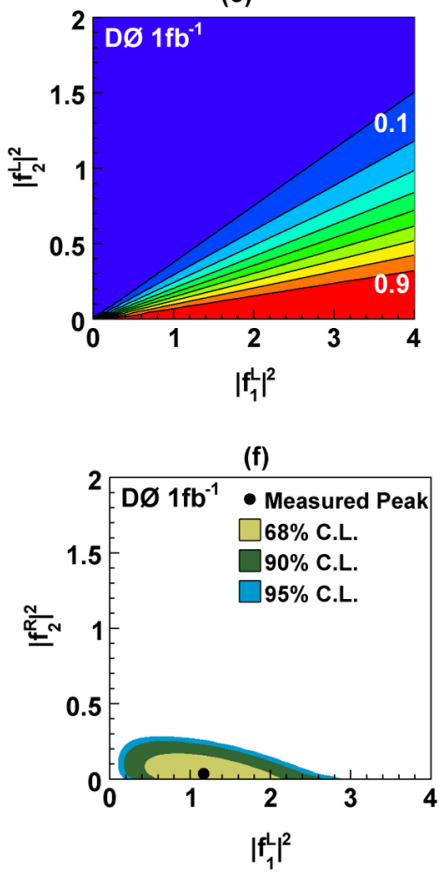

(d)

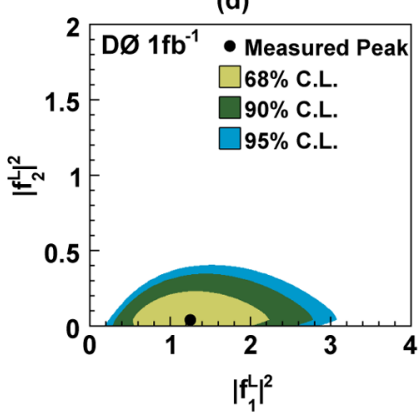

FIG. 1 (color online). $\quad W$ helicity prior (a), (c), (e) and final posterior density (b), (d), (f) for right- vs left-handed vector coupling (a) and (b), left-handed tensor vs left-handed vector coupling (c) and (d), and right-handed tensor vs left-handed vector coupling (e) and (f). The $W$ helicity prior is normalized to a peak value of one and shown as equally spaced contours between zero and one. The posterior density is shown as contours of equal probability density. 
TABLE I. Measured values with uncertainties and upper limits at the $95 \%$ C.L. for $W t b$ couplings in three different scenarios.

\begin{tabular}{ccc}
\hline \hline Scenario & \multicolumn{1}{c}{ Coupling } & Coupling limit if $f_{1}^{L}=1$ \\
\hline$\left(L_{1}, R_{1}\right)$ & $\left|f_{1}^{L}\right|^{2}=1.27_{-0.48}^{+0.57}$ & \\
& $\left|f_{1}^{R}\right|^{2}<0.95$ & $\left|f_{1}^{R}\right|^{2}<1.01$ \\
$\left(L_{1}, L_{2}\right)$ & $\left|f_{1}^{L}\right|^{2}=1.27_{-0.48}^{+0.60}$ & \\
& $\left|f_{2}^{L}\right|^{2}<0.32$ & $\left|f_{2}^{L}\right|^{2}<0.28$ \\
$\left(L_{1}, R_{2}\right)$ & $\left|f_{1}^{L}\right|^{2}=1.04_{-0.49}^{+0.55}$ & \\
& $\left|f_{2}^{R}\right|^{2}<0.23$ & $\left|f_{2}^{R}\right|^{2}<0.23$ \\
\hline \hline
\end{tabular}

(i.e., if the final selection efficiency changes) and by modifying the shape of the $\cos \theta^{\star}$ templates. Systematic uncertainties in the single top analysis arise from the $W+$ jets normalization, the $W+$ jets flavor composition estimate, and the top-quark pair background modeling.

Most of the systematic uncertainties are taken to be $100 \%$ correlated between the two analyses. Systematic uncertainties that affect only the $W$ helicity analysis are MC statistics and MC background model. Systematic uncertainties arising from the luminosity measurement affect only the single top analysis.

We use a Bayesian statistical analysis [20] to combine the $W$ helicity result with the single top anomalous coupling result. The likelihood result from the $W$ helicity analysis is used as a prior to the single top anomalous coupling analysis.

For any pair of values of the two coupling form factors under consideration, we compare the boosted decision tree output for the data with the sum of backgrounds and the two signals. In the scenario where $f_{1}^{L}$ and $f_{2}^{L}$ are nonzero, the two amplitudes interfere, which we take into account by using a superposition of three signal samples: one with only left-handed vector couplings; one with only lefthanded tensor couplings; and one with both coupling form factors set to one, containing the interference term. We then compute a likelihood as a product over all separate analysis channels. We assume Poisson distributions for the observed counts and use multivariate Gaussian distributions to model the uncertainties on the combined signal acceptance and background yields, including correlations. The uncertainties are evaluated through MC integration. We generate an ensemble of 5000 samples, each with a different shift in the various systematic uncertainties, and compute the Bayesian posterior for each sample. The final posterior is then the ensemble average of all individual posteriors.

The two-dimensional posterior probability density is computed as a function of $\left|f_{1}^{L}\right|^{2}$ and $\left|f_{X}\right|^{2}$, where $f_{X}$ is $f_{1}^{R}, f_{2}^{L}$, or $f_{2}^{R}$. These probability distributions are shown in Fig. 1. In all three scenarios, we measure approximately zero for the anomalous coupling form factors and favor the left-handed vector hypothesis over the alternative hypothesis. We compute 95\% Confidence Level (C.L.) upper limits on these form factors by integrating out the left-handed vector coupling form factor to get a one-dimensional posterior probability density. The measured values are given in Table I.

In comparison, the limits at 95\% C.L. without the $W$ helicity constraints are $\left|f_{1}^{R}\right|^{2}<1.83,\left|f_{2}^{L}\right|^{2}<0.52$, and $\left|f_{2}^{R}\right|^{2}<0.24$. The kinematic distributions of the $f_{1}^{L}$ and $f_{1}^{R}$ single top-quark samples are similar enough that the single top anomalous coupling analysis in this scenario is mainly sensitive to the total cross section. Hence, the $W$ helicity analysis improves the $\left|f_{1}^{R}\right|^{2}$ limit significantly. Conversely, it does not add much information to the right-tensor coupling limit where most of the sensitivity is provided by the single top anomalous coupling analysis.

In summary, we have presented the first study of Wtb couplings that combines $W$ helicity measurements in topquark decay with anomalous couplings searches in the single top-quark final state, thus using all applicable topquark measurements by D0. We find consistency with the SM and set 95\% C.L. limits on anomalous $W t b$ couplings. Our limits represent significant improvements over previous results, and rule out a right-handed top-quark vector coupling form factor of magnitude one for the first time.

We thank the staffs at Fermilab and collaborating institutions, and acknowledge support from the DOE and NSF (USA); CEA and CNRS/IN2P3 (France); FASI, Rosatom and RFBR (Russia); CNPq, FAPERJ, FAPESP, and FUNDUNESP (Brazil); DAE and DST (India); Colciencias (Colombia); CONACyT (Mexico); KRF and KOSEF (Korea); CONICET and UBACyT (Argentina); FOM (The Netherlands); STFC (United Kingdom); MSMT and GACR (Czech Republic); CRC Program, CFI, NSERC, and WestGrid Project (Canada); BMBF and DFG (Germany); SFI (Ireland); The Swedish Research Council (Sweden); CAS and CNSF (China); and the Alexander von Humboldt Foundation (Germany).

*Visitor from Augustana College, Sioux Falls, SD, USA

${ }^{\dagger}$ Visitor from Rutgers University, Piscataway, NJ, USA

*Visitor from The University of Liverpool, Liverpool, UK

${ }^{\S}$ Visitor from II. Physikalisches Institut, Georg-AugustUniversity, Göttingen, Germany

"Visitor from Centro de Investigacion en ComputacionIPN, Mexico City, Mexico

"Visitor from ECFM, Universidad Autonoma de Sinaloa, Culiacán, Mexico

**Visitor from Helsinki Institute of Physics, Helsinki, Finland

${ }^{\dagger \dagger}$ Visitor from Universität Bern, Bern, Switzerland

Visitor from Universität Zürich, Zürich, Switzerland

${ }^{\S}$ Deceased

[1] T. M.P Tait and C.-P. Yuan, Phys. Rev. D 63, 014018 (2000). 
[2] G. L. Kane, G. A. Ladinsky, and C.-P. Yuan, Phys. Rev. D 45, 124 (1992).

[3] N. Cabibbo, Phys. Rev. Lett. 10, 531 (1963); M. Kobayashi and T. Maskawa, Prog. Theor. Phys. 49, 652 (1973).

[4] F. Larios, M. A. Perez, and C.-P. Yuan, Phys. Lett. B 457, 334 (1999); G. Burdman, M. C. Gonzalez-Garcia, and S. F. Novaes, Phys. Rev. D 61, 114016 (2000), and references therein.

[5] V. M. Abazov et al. (D0 Collaboration), Nucl. Instrum. Methods Phys. Res., Sect. A 565, 463 (2006).

[6] V. M. Abazov et al. (D0 Collaboration), Phys. Rev. Lett. 100, 062004 (2008).

[7] V. M. Abazov et al. (D0 Collaboration), Phys. Rev. Lett. 101, 221801 (2008).

[8] C. R. Chen, F. Larios, and C. P. Yuan, Phys. Lett. B 631, 126 (2005).

[9] M. L. Mangano et al., J. High Energy Phys. 07 (2003) 001. We used ALPGEN version 2.05.

[10] T. Sjöstrand et al., arXiv:hep-ph/0308153. We used PYTHIA version 6.323 .

[11] J. Pumplin et al., J. High Energy Phys. 07 (2002) 012; D. Stump et al., ibid. 10 (2003) 046.
[12] R. Brun and F. Carminati, CERN Program Library Long Writeup Report No. W5013, 1993 (unpublished).

[13] V. M. Abazov et al. (D0 Collaboration), Phys. Rev. Lett. 98, 181802 (2007); V.M. Abazov et al. (D0 Collaboration), Phys. Rev. D 78, 012005 (2008).

[14] T. Aaltonen et al. (CDF Collaboration), Phys. Rev. Lett. 101, 252001 (2008).

[15] Z. Sullivan, Phys. Rev. D 70, 114012 (2004).

[16] E. Boos et al., Phys. At. Nucl. 69, 1317 (2006); E. Boos et al. (CompHEP Collaboration), Nucl. Instrum. Methods Phys. Res., Sect. A 534, 250 (2004).

[17] N. Kidonakis and R. Vogt, Phys. Rev. D 68, 114014 (2003).

[18] L. Breiman et al., Classification and Regression Trees (Wadsworth, Stamford, 1984).

[19] Y. Freund and R.E. Schapire, in Machine Learning: Proceedings of the Thirteenth International Conference, edited by L. Saitta (Morgan Kaufmann, San Francisco, 1996), p. 148.

[20] I. Bertram et al., Report No. FERMILAB-TM-2104, 2000. 